\title{
Fitting formulae of the reduced-shear power spectrum for weak lensing ${ }^{\star}$ (Research Note)
}

\author{
M. Kilbinger ${ }^{1,2,3}$ \\ ${ }^{1}$ Excellence Cluster Universe, Technische Universität München, Boltzmannstr. 2, 85748 Garching, Germany \\ e-mail: martin.kilbinger@universe-cluster.de \\ 2 Universitäts-Sternwarte München, Scheinerstr. 1, 81679 München, Germany \\ 3 Shanghai Key Lab for Astrophysics, Shanghai Normal University, Shanghai 200234, PR China
}

Received 20 April 2010 / Accepted 9 June 2010

\section{ABSTRACT}

\begin{abstract}
Context. Weak gravitational lensing is a powerful probe of large-scale structure and cosmology. Most commonly, second-order correlations of observed galaxy ellipticities are expressed as a projection of the matter power spectrum, corresponding to the lowest-order approximation between the projected and the three-dimensional power spectrum.

Aims. The dominant lensing-only contribution beyond the zero-order approximation is the reduced shear, which takes into account not only lensing-induced distortions but also the isotropic magnification of galaxy images. This involves an integral over the matter bispectrum. We provide a fast and general way to calculate this correction term.

Methods. Using a model for the matter bispectrum, we fit elementary functions to the reduced-shear contribution and its derivatives with respect to cosmological parameters. The dependence on cosmology is encompassed in a Taylor-expansion around a fiducial model.

Results. Within a region in parameter space comprising the WMAP7 68\% error ellipsoid, the total reduced-shear power spectrum (shear plus fitted reduced-shear correction) is accurate to $1 \%(2 \%)$ for $\ell<10^{4}\left(\ell<2 \times 10^{5}\right)$. This corresponds to a factor of four reduction in the bias compared to the case where no correction is used. This precision is necessary to match the accuracy of current non-linear power spectrum predictions from numerical simulations.
\end{abstract}

Key words. gravitational lensing: weak - large-scale structure of Universe

\section{Introduction}

Cosmic shear surveys exploit the distortions of distant galaxy images induced by the matter structures in the Universe on large scales. Weak cosmological lensing has become an important tool to measure cosmological parameters. The current precision in the measured shapes of high-redshift galaxies is at the few percent level providing important constraints on the matter density $\Omega_{\mathrm{m}}$ and the power-spectrum normalisation $\sigma_{8}$. Future surveys aim to achieve sub-percent level accuracy in measuring darkenergy properties or deviations from general relativity.

To reach this precision for cosmological parameters, efforts not only on the observational but also on the theoretical side have to be made. To predict the shear power spectrum from theoretical models, several approximations are usually made to facilitate calculations. When comparing and fitting models to observations, these simplifications can significantly bias the resulting cosmological parameters. One of these approximations is to neglect the correction due to the so-called reduced shear.

The observed ellipticity of galaxies is an estimator of the reduced shear

$$
g=\frac{\gamma}{1-\kappa}
$$

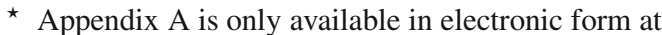
http://www . aanda.org
}

where $\gamma$ is the shear and $\kappa$ is the convergence. Here and in the following, both shear and reduced shear are represented as complex quantities, $\gamma=\gamma_{1}+\mathrm{i} \gamma_{2}, g=g_{1}+\mathrm{i} g_{2}$. Since in weak lensing the convergence is small, $|\kappa| \ll 1$, one usually simplifies the above relation to linear order, $g \approx \gamma$.

The effect of reduced shear was examined using $N$-body simulations by White (2005) and compared with theoretical predictions in Dodelson et al. (2006). Corrections to the lensing skewness were discussed in Schneider et al. (1998) and Dodelson \& Zhang (2005). An extensive study of higher-order corrections to the weak-lensing power spectrum, including reduced shear, was presented by Krause \& Hirata (2009). Compared with corrections due to light-path integration (relaxation of the Born approximation) and lens-lens coupling (e.g., Seitz 1993; Bernardeau et al. 1997), reduced-shear corrections represent the dominant contribution to the weak-lensing power spectrum. The lowest-order correction term, which is of order $O\left(\phi^{3}\right)$ in the gravitational potential $\phi$, reaches $10 \%$ of the total lensing spectrum. This is the term that we consider in this work. The nextorder correction at $O\left(\phi^{4}\right)$ is one magnitude below the $O\left(\phi^{3}\right)$-term (Krause \& Hirata 2009). Ignoring reduced shear will cause a bias in cosmological parameters, which is comparable to the statistical error from future surveys like $\mathrm{DES}^{1}, \mathrm{LSST}^{2}$, or Euclid ${ }^{3}$

\footnotetext{
1 http://www . darkenergysurvey .org

2 http://www. lsst.org

3 http://www.ias.u-psud.fr/imEuclid
} 
(Shapiro 2009). For the weak-lensing results of the COSMOS survey ${ }^{4}$, an estimate from numerical simulations yielded a $1 \%$ underestimation of $\sigma_{8}$ (Schrabback et al. 2010).

Another correction to weak lensing from a magnitudelimited galaxy sample is the so-called magnification bias. Hamana (2001) found its contribution to the lensing power spectrum to be small. The corresponding third-order correction terms were also calculated and compared to numerical simulations by Ménard et al. (2003). However, if not corrected for, future surveys will yield significantly biased cosmological parameter constraints (Schmidt et al. 2009a). The lowest-order correction term has the same functional form as the reduced-shear one, and therefore both can be dealt with simultaneously. Higher-order terms contribute by smaller than one percent to the lensing power spectrum (Krause \& Hirata 2009).

There are additional important sources of contamination to the weak lensing observables, some of which may be larger than the reduced-shear corrections. These involve galaxy clustering, the intrinsic alignment of galaxies with each other or with the surrounding dark matter haloes, and the modelling of the non-linear dark-matter power spectrum and baryonic processes. The modelling of these effects requires detailed understanding of baryonic physics, galaxy formation, the interplay between galaxies and dark matter, and massive numerical simulations in the case of the non-linear power spectrum. In contrast, taking into account the reduced shear is straightforward and can be done with sufficient accuracy to render it a sub-dominant contamination.

In the next section, we review the expressions for the lowestorder reduced-shear term, and introduce a fitting scheme to efficiently model this term. In Sect. 3, we present our results, demonstrating the accuracy of the fits. We conclude this paper in Sect. 4.

\section{Reduced shear}

\subsection{Lowest-order expansion}

For small values of the convergence, $|\kappa|<1$, the reduced shear (1) is expanded as

$g=\frac{\gamma}{1-\kappa}=\gamma\left[1+\kappa+\kappa^{2}+\ldots\right]$

To calculate the power spectrum of the reduced shear $g$, we apply a Fourier transform to Eq. (2) to second order

$\hat{g}(\boldsymbol{\ell}) \approx \hat{\gamma}(\boldsymbol{\ell})+(\hat{\gamma} * \hat{\kappa})(\boldsymbol{\ell})$

In our notation, the convolution of $\hat{\gamma}$ and $\hat{\kappa}$ is

$(\hat{\gamma} * \hat{\kappa})(\boldsymbol{\ell})=\int \frac{\mathrm{d}^{2} \ell^{\prime}}{(2 \pi)^{2}} \hat{\gamma}\left(\boldsymbol{\ell}^{\prime}\right) \hat{\kappa}\left(\boldsymbol{\ell}-\boldsymbol{\ell}^{\prime}\right)$

We assume that the reduced shear is measured in redshift bins. With $P_{g}^{m n}$, we denote the reduced-shear (cross-)power spectrum for two redshift bins with indices $m$ and $n$. In general, the shear field can be decomposed into an E-mode (gradient) and a $\mathrm{B}$-mode (curl). However, as is the case for cosmic shear at lowest order, the reduced-shear correction of order $O\left(\phi^{3}\right)$ does not have a B-mode component (Schneider et al. 2002), and in the following we focus only on the E-mode. In the absence of a B-mode, the reduced-shear power spectrum is defined to be (Schneider et al. 2002)

$$
\left\langle\hat{g}^{m}(\boldsymbol{\ell}) \hat{g}^{n *}\left(\boldsymbol{\ell}^{\prime}\right)\right\rangle=(2 \pi)^{2} \delta_{\mathrm{D}}\left(\boldsymbol{\ell}-\boldsymbol{\ell}^{\prime}\right) P_{g}^{m n}(\ell),
$$

${ }^{4}$ http://cosmos.astro. caltech.edu where $g^{m}$ denotes the reduced shear measured from galaxies in the $m$ th redshift bin, the asterisk $\left(^{*}\right)$ denotes complex conjugation, and $\delta_{\mathrm{D}}$ is the Dirac delta-function.

The reduced-shear power spectrum is the sum of the convergence power spectrum (which equals the shear power spectrum) and a correction given by

$P_{g}^{m n}(\ell) \approx P_{\kappa}^{m n}(\ell)+P_{g}^{(1)^{m n}}(\ell)$

$(2 \pi)^{2} \delta_{\mathrm{D}}\left(\boldsymbol{\ell}-\boldsymbol{\ell}^{\prime}\right) P_{g}^{(1)^{m n}}(\ell)=\left\langle\hat{\gamma}^{m}(\boldsymbol{\ell})\left(\hat{\gamma}^{n} * \hat{\kappa}^{n}\right)^{*}\left(\boldsymbol{\ell}^{\prime}\right)\right\rangle+(m \leftrightarrow n)$.

Using the relation between shear and convergence, $\hat{\gamma}(\boldsymbol{\ell})=$ $\exp (2 \mathrm{i} \beta) \hat{\kappa}(\boldsymbol{\ell})$, where $\beta$ is the polar angle of the wave vector $\boldsymbol{\ell}$, and that the convergence is a real field, $\hat{\kappa}^{*}(\boldsymbol{\ell})=\hat{\kappa}(-\boldsymbol{\ell})$, the third-order correlator in Eq. (7) can be written in terms of the convergence bispectrum $B_{\kappa}$, which is defined by the equation

$\left\langle\hat{\kappa}^{m}\left(\boldsymbol{\ell}_{1}\right) \hat{\kappa}^{n}\left(\boldsymbol{\ell}_{2}\right) \hat{\kappa}^{p}\left(\boldsymbol{\ell}_{3}\right)\right\rangle=(2 \pi)^{2} \delta_{\mathrm{D}}\left(\boldsymbol{\ell}_{1}+\boldsymbol{\ell}_{2}+\boldsymbol{\ell}_{3}\right) B_{\kappa}^{m n p}\left(\boldsymbol{\ell}_{1}, \boldsymbol{\ell}_{2}\right)$.

With this, we derive the correction term

$P_{g}^{(1) m n}(\ell)=\int \frac{\mathrm{d}^{2} \ell^{\prime}}{(2 \pi)^{2}} \mathrm{e}^{-2 \mathrm{i} \beta^{\prime}}\left[B_{\kappa}^{m n n}\left(\boldsymbol{\ell}_{0},-\boldsymbol{\ell}^{\prime}\right)+B_{\kappa}^{n m m}\left(\boldsymbol{\ell}_{0},-\boldsymbol{\ell}^{\prime}\right)\right]$.

Without loss of generality, we have set $\beta=0$ and introduced $\boldsymbol{\ell}_{0}=(\ell, 0)$. In the integrand, $\beta^{\prime}$ is the polar angle of $\boldsymbol{\ell}^{\prime}$.

The convergence bispectrum can be expressed in terms of the three-dimensional matter bispectrum $B_{\delta}$ using Limber's equation (Limber 1953; Kaiser 1992) providing

$$
\begin{aligned}
& B_{\kappa}^{m n p}\left(\boldsymbol{\ell}_{1}, \boldsymbol{\ell}_{2}\right)=\int_{a_{\mathrm{lim}}}^{1} \mathrm{~d} a F^{n m p}(a) B_{\delta}\left(\frac{\boldsymbol{\ell}_{1}}{f_{K}[\chi(a)]}, \frac{\boldsymbol{\ell}_{2}}{f_{K}[\chi(a)]} ; \chi(a)\right) \\
& F^{m n p}(a)=\frac{\mathrm{d} \chi}{\mathrm{d} a} \frac{G^{m}(a) G^{n}(a) G^{p}(a)}{f_{K}[\chi(a)]}
\end{aligned}
$$

The lower limit of the integral over the scale factor $a$ corresponds to the limiting redshift, $a_{\lim }=1 /\left(1+z_{\mathrm{lim}}\right), \chi$ is the comoving distance, and $f_{K}$ is the comoving angular diameter distance. The lensing efficiency $G^{m}$ for the $m$ th redshift bin is

$$
G^{m}(a)=\frac{3}{2}\left(\frac{H_{0}}{c}\right)^{2} \frac{\Omega_{\mathrm{m}}}{a} \int_{a_{\mathrm{lim}}}^{a} \mathrm{~d} a^{\prime} p_{m}\left(a^{\prime}\right) \frac{f_{K}\left[\chi\left(a^{\prime}\right)-\chi(a)\right]}{f_{K}\left[\chi\left(a^{\prime}\right)\right]},
$$

which includes an integral over the probability distribution $p_{m}$ of the number density of source galaxies in the $m$ th bin.

\subsection{Expansion around a fiducial model}

In the expressions needed to calculate the first-order reducedshear power spectrum in Eqs. $(9,10)$, we can interchange the $\ell$ and the $a$-integration, and replace the latter by a finite sum. Then

$$
\begin{aligned}
& P_{g}^{(1) m n}(\ell) \approx \Delta a \sum_{k=1}^{N_{a}} \bar{F}^{m n}\left(a_{k}\right) Q\left(\ell, a_{k}\right) \\
& \bar{F}^{m n}(a)=\frac{1}{2}\left[F^{m n n}\left(a_{k}\right)+F^{n m m}\left(a_{k}\right)\right] \\
& Q(\ell, a)=2 \int \frac{\mathrm{d}^{2} \ell^{\prime}}{(2 \pi)^{2}} \cos \left(2 \beta^{\prime}\right) B_{\delta}\left(\frac{\ell_{0}}{f_{K}[\chi(a)]}, \frac{-\ell^{\prime}}{f_{K}[\chi(a)]}\right) .
\end{aligned}
$$

Evaluating the $\ell^{\prime}$-integral is not a difficult but very timeconsuming numerical problem. It is significantly slower than the calculation of the matter power spectrum necessary for the convergence power spectrum. This makes reduced-shear corrections the limiting factor in the parameter analysis of a weaklensing survey, for example using Monte Carlo sampling. In the 
Table 1. Parameter limits for where the accuracy of the fitted reducedshear power spectrum is better than $1 \%$ for $\ell<10^{4}$, and better than $2 \%$ for $\ell<2 \times 10^{5}$ (see also Fig. 3).

\begin{tabular}{llll}
\hline \hline$\alpha$ & Parameter & Lower & Upper \\
\hline 1 & $\Omega_{\mathrm{m}}$ & 0.22 & 0.35 \\
2 & $\Omega_{\mathrm{de}}$ & 0.33 & 1.03 \\
3 & $w$ & -1.6 & -0.6 \\
4 & $\Omega_{\mathrm{b}}$ & 0.005 & 0.085 \\
5 & $h$ & 0.61 & 1.11 \\
6 & $\sigma_{8}$ & 0.65 & 0.93 \\
7 & $n_{\mathrm{s}}$ & 0.86 & 1.16 \\
\hline
\end{tabular}

Notes. Note that for each given range, the other parameters were kept fixed.

following, we present fitting formulae that provide an accurate approximation for this term within a region in parameter space around a fiducial cosmological model. The $\ell$-independent term $F^{m n n}(a)$ is easily computed and can be tabulated. Since it depends on the source redshift distribution, it has to be determined for each survey.

We perform a Taylor-expansion to first order $P^{(1)^{m n}}$ as a function of a cosmological parameter vector $\boldsymbol{p}=\left(p_{1}, p_{2}, \ldots, p_{M}\right)$ around a fiducial cosmological model with parameter $\boldsymbol{p}_{0}$

$P_{g}^{(1)^{m n}}(\ell, \boldsymbol{p}) \approx P_{g}^{(1)^{m n}}\left(\ell, \boldsymbol{p}_{0}\right)+\left.\left(\boldsymbol{p}-\boldsymbol{p}_{0}\right) \boldsymbol{\nabla}_{\boldsymbol{p}} P_{g}^{(1)^{m n}}(\ell, \boldsymbol{p})\right|_{\boldsymbol{p}=\boldsymbol{p}_{0}}$.

Inserting Eq. (12), the derivatives with respect to cosmological parameters are given by

$$
\begin{aligned}
\frac{\partial P_{g}^{(1)}(\ell n}{\partial p_{\alpha}}= & \Delta a \sum_{k=1}^{N_{a}}\left\{\bar{F}^{m n}\left(a_{k}, \boldsymbol{p}\right) \frac{\partial Q\left(\ell, a_{k}, \boldsymbol{p}\right)}{\partial p_{\alpha}}\right. \\
& \left.+\frac{\partial \bar{F}^{m n}\left(a_{k}, \boldsymbol{p}\right)}{\partial p_{\alpha}} Q\left(\ell, a_{k}, \boldsymbol{p}\right)\right\} .
\end{aligned}
$$

In Sect. 3, we consider the cosmological parameters $\boldsymbol{p}=$ $\left(\Omega_{\mathrm{m}}, \Omega_{\mathrm{de}}, w, \Omega_{\mathrm{b}}, h, \sigma_{8}, n_{\mathrm{s}}\right)$ (see also Table 1$)$.

\subsection{Magnification and size bias}

A typical galaxy sample used in weak cosmological lensing is selected by both magnitude and galaxy size. Magnification due to lensing changes both quantities (e.g. Bartelmann \& Schneider 2001), and therefore introduces a correlation between number density and convergence. If the number density of galaxies with fluxes higher than some flux $S$, and sizes larger than some size $R$ can be written as power laws,

$p(>S,>R) \propto S^{-\alpha} R^{-\beta}$,

the observed number density differs from the unlensed one $p_{0}$ to first order, becoming

$p=p_{0}(1+q \kappa) ; \quad q=2(\alpha+\beta-1)$,

according to Schmidt et al. (2009a,b). This simple linear model assumes that the galaxy selection function depends on flux and size in a separable way. We refer to Wyithe et al. (2003) for a generalisation that takes into account correlations between the two quantities.

Magnification and size bias induces a lowest-order correction to the lensing power spectrum which has the same form as

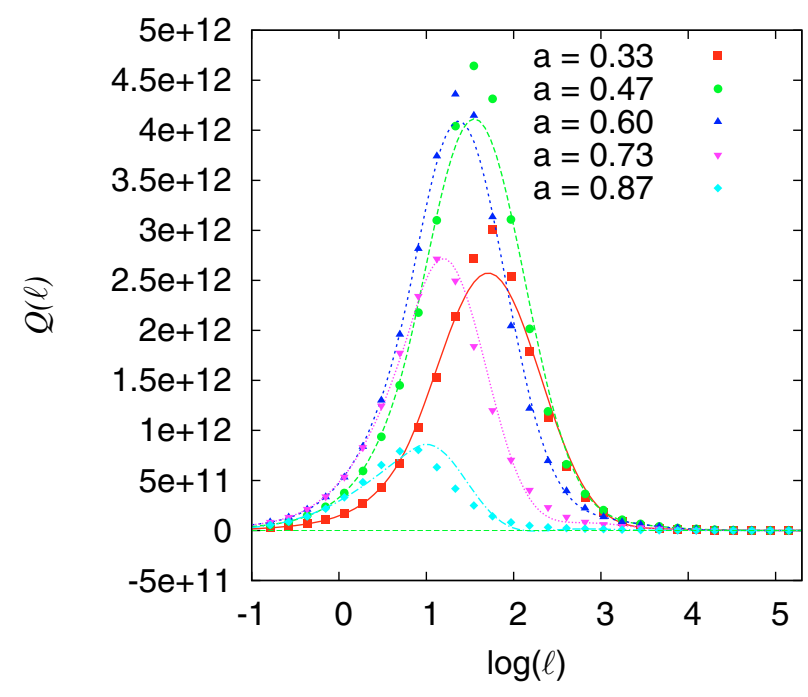

Fig. 1. The function $Q^{(0)}(\ell)=Q(\ell)$ for different scale factors $a$ as indicated in the panel (points), and the composite fitting function (Eq. (21), lines).

for reduced shear. Therefore, we can add the corresponding correction term $P_{\mu}^{(1)^{m n}}$ to Eq. (6) with

$P_{\mu}^{(1)}{ }_{\mu}^{m n}(\ell) \approx \Delta a \sum_{k=1}^{N_{a}} \bar{F}^{m n}\left(a_{k}\right) Q_{\mu}\left(\ell, a_{k}\right) ;$

$Q_{\mu}(\ell, a)=q Q(\ell, a)$,

where the function $Q$ is given in Eq. (12).

\subsection{Fitting formulae}

For simplicity, we define $Q^{(0)}=Q$ and $Q^{(\alpha)}=\partial Q / \partial p_{\alpha}$ for $\alpha=1, \ldots, M$. These correspond to the $\ell$-dependent terms in Eqs. (12) and (14), which behave as near-power laws for both small and large $\ell$. With the abbreviation $y=\ln \ell$, we perform two linear fits of $\ln \left|Q^{(\alpha)}(y)\right|$ for $y<y_{\text {low }}=3$ and $y>y_{\text {up }}=$ 11.5 , respectively. We smoothly piece together these two asymptotic functions with a cubic spline $s^{\alpha}(y)$ such that the composite function $\ln h^{(\alpha)}(y), \alpha=0, \ldots, M$, and its first derivative are continuous

$\ln h^{(\alpha)}(y, a)=\left\{\begin{array}{lll}g_{\mathrm{low}}^{(\alpha)}(y, a) & \text { for } \quad y<y_{\text {low }} \\ s^{(\alpha)}(y, a) & \text { for } \quad y_{\text {low }} \leq y \leq y_{\text {up }} \\ g_{\mathrm{up}}^{(\alpha)}(y, a) & \text { for } \quad y>y_{\mathrm{up}} ;\end{array}\right.$

for the linear functions

$g_{\mathrm{low}}^{(\alpha)}(y, a)=b_{0}^{(\alpha)}(a) \cdot y+b_{1}^{(\alpha)}(a) ;$

$g_{\mathrm{up}}^{(\alpha)}(y, a)=b_{2}^{(\alpha)}(a) \cdot y+b_{3}^{(\alpha)}(a)$,

and the cubic spline

$s^{(\alpha)}(y, a)=\sum_{v=0}^{3} r_{v}^{(\alpha)}(a) \cdot\left(\frac{y-y_{\mathrm{low}}}{\Delta}\right)^{v} ;$

$\Delta=y_{\text {up }}-y_{\text {low }}$;

$r_{0}^{(\alpha)}(a)=g_{\mathrm{low}}^{(\alpha)}\left(y_{\mathrm{low}}, a\right)$

$r_{1}^{(\alpha)}(a)=\Delta \cdot b_{0}^{(\alpha)}(a)$

$r_{2}^{(\alpha)}(a)=3 g_{\mathrm{up}}^{(\alpha)}\left(y_{\mathrm{up}}, a\right)-\Delta \cdot b_{2}^{(\alpha)}(a)-3 r_{0}^{(\alpha)}(a)-2 r_{1}^{(\alpha)}(a) ;$

$r_{3}^{(\alpha)}(a)=\Delta \cdot b_{2}^{(\alpha)}(a)-2 g_{\mathrm{up}}^{(\alpha)}\left(y_{\mathrm{up}}, a\right)+2 r_{0}^{(\alpha)}(a)+r_{1}^{(\alpha)}(a)$. 
$Q$

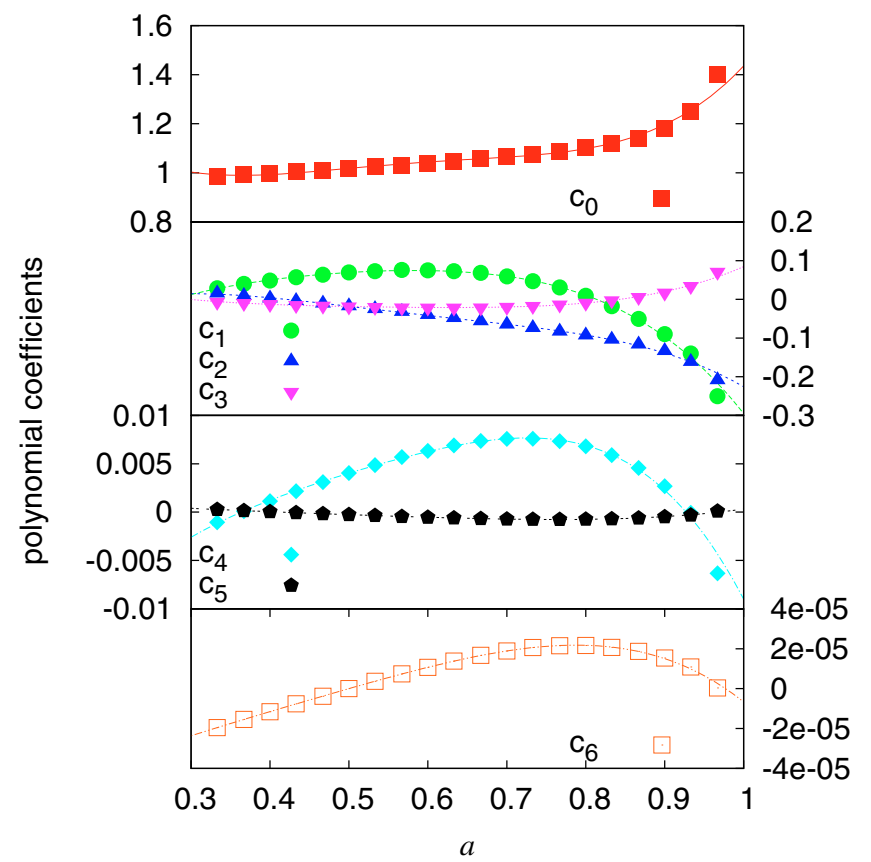

$Q$
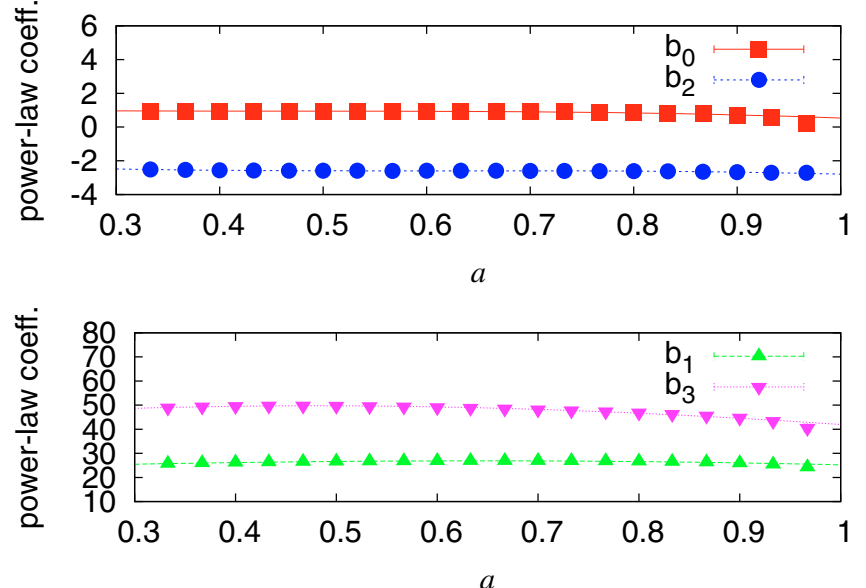

Fig. 2. Fit coefficients as a function of scale factor. Left: the polynomial fit coefficients $c_{i}^{(0)}$ for $i=0, \ldots, N=6$ (Eq. (21)). The lines are polynomials in $a$ or order $N_{c}=3$. Right: the power-law coefficients $b_{i}^{(0)}, i=0, \ldots, 3$ of the composite function $h^{(0)}$ (Eq. (18)). The lines are polynomials in $a$ of order $N_{b}=3$.

The ratios $Q^{(\alpha)} / h^{(\alpha)}$ are then fitted by polynomials of order $N$ in $y=\ln \ell$, at the fiducial cosmology $\boldsymbol{p}=\boldsymbol{p}_{0}$, and for each scale factor $a=a_{k}, k=1, \ldots, N_{a}$,

$Q^{(\alpha)}(y, a) \approx h^{(\alpha)}(y, a) \cdot \sum_{i=0}^{N} c_{i}^{(\alpha)}(a) y^{i} ; \quad \alpha=0, \ldots, M$.

We note that we cannot fit the logarithm of the functions $Q^{(\alpha)}$, since the derivatives of $Q$ with respect to some cosmological parameters change sign.

The fit coefficients $b_{i}^{(\alpha)}, c_{i}^{(\alpha)}$ are expected to smoothly vary with the scale factor $a$. We therefore perform another series of fits by polynomials of order $N_{b}$ and $N_{c}$, respectively

$b_{i}^{(\alpha)}(a) \approx \sum_{j=0}^{N_{b}} B_{i j}^{(\alpha)} a^{j} ; c_{i}^{(\alpha)}(a) \approx \sum_{j=0}^{N_{c}} C_{i j}^{(\alpha)} a^{j}$.

The two matrices $B^{(\alpha)}$ and $C^{(\alpha)}$ for $\alpha=0, \ldots, M$ defined in the last equation completely determine our approximation for the $\ell$-dependent terms of the reduced-shear power spectrum correction.

\section{Results}

We use a fiducial flat $\Lambda$ CDM cosmological model with WMAP7-like parameters, $\Omega_{\mathrm{m}}=0.27, \Omega_{\mathrm{b}}=0.045, h=$ $0.71, \sigma_{8}=0.8$ and $n_{\mathrm{s}}=0.96$ (Komatsu et al. 2010). The dark-matter bispectrum is calculated according to Scoccimarro \& Couchman (2001). Their fitting formula is accurate to only $30-50 \%$ on small scales; this is however sufficient for our purpose. We note also that the bispectrum is not calibrated for any dark-energy model other than $\Lambda \mathrm{CDM}$. For the matter power spectrum, we use the "halofit" fitting formula of Smith et al. (2003) and the transfer function "shape fit" from
Eisenstein \& $\mathrm{Hu}$ (1998). Following the icosmo.org code (Refregier et al. 2008) for models with $w \neq-1$, we modify "halofit" to interpolate between $\Lambda \mathrm{CDM}$ and $w=-1 / 3$, which behaves in a similar way to an OCDM model (for more details, see Schrabback et al. 2010).

The function $Q$ and the corresponding fit with the composite function given in Eq. (21) are shown in Fig. 1. The polynomial that is part of the composite function has order $N=6$. The fitting coefficients $b_{i}^{(0)}$ and $c_{i}^{(0)}$ are plotted in Fig. 2. Although the higher-order polynomial coefficients have relatively low amplitudes (right panel), we found that a polynomial of order 6 is necessary to provide a good fit to the reduced-shear power spectrum, as discussed below. For the polynomial fits of the coefficients as functions of $a$ (22), we chose $N_{b}=N_{c}=3$. These cubic polynomials provide sufficient accuracy, in particular for $a \rightarrow 1$, where the coefficients show the most variation. This is important because the reduced-shear correction spectrum in Eq. (12) obtains a large contribution from large $a$.

We perform the fits in the $\ell$-range between 0.1 and $2 \times 10^{5}$. The functions $Q^{(\alpha)}$ are not perfect power laws, therefore the fit for large $\ell$ is not excellent. We found an improvement of our final results for the total power spectrum by adding 0.05 to $b_{2}$ after performing the fits.

The fitting functions for the reduced-shear power spectrum corrections provide accurate results over a wide range in $\ell$. We illustrate the case of a single redshift bin with distribution $p(z)=\left(z / z_{0}\right)^{\alpha} \cdot \exp \left[-\left(z / z_{0}\right)^{\beta}\right]$ and parameters $\alpha=2, \beta=1.5$, and $z_{0}=0.5$. The maximum considered redshift is $z_{\max }=2$, which results in a mean redshift of 0.75 . At $\ell=10^{3}$ the reduced-shear correction to the convergence power spectrum starts to become important. On smaller scales, the latter underestimates the total power spectrum by more than $1 \%$. For $10^{3}<\ell<10^{5}$, we fit $P_{\mathrm{g}}^{(1)}$ (Eq. (7)) to better than $20 \%$. This is sufficient to provide an approximation of the total power spectrum at the percent-level. 

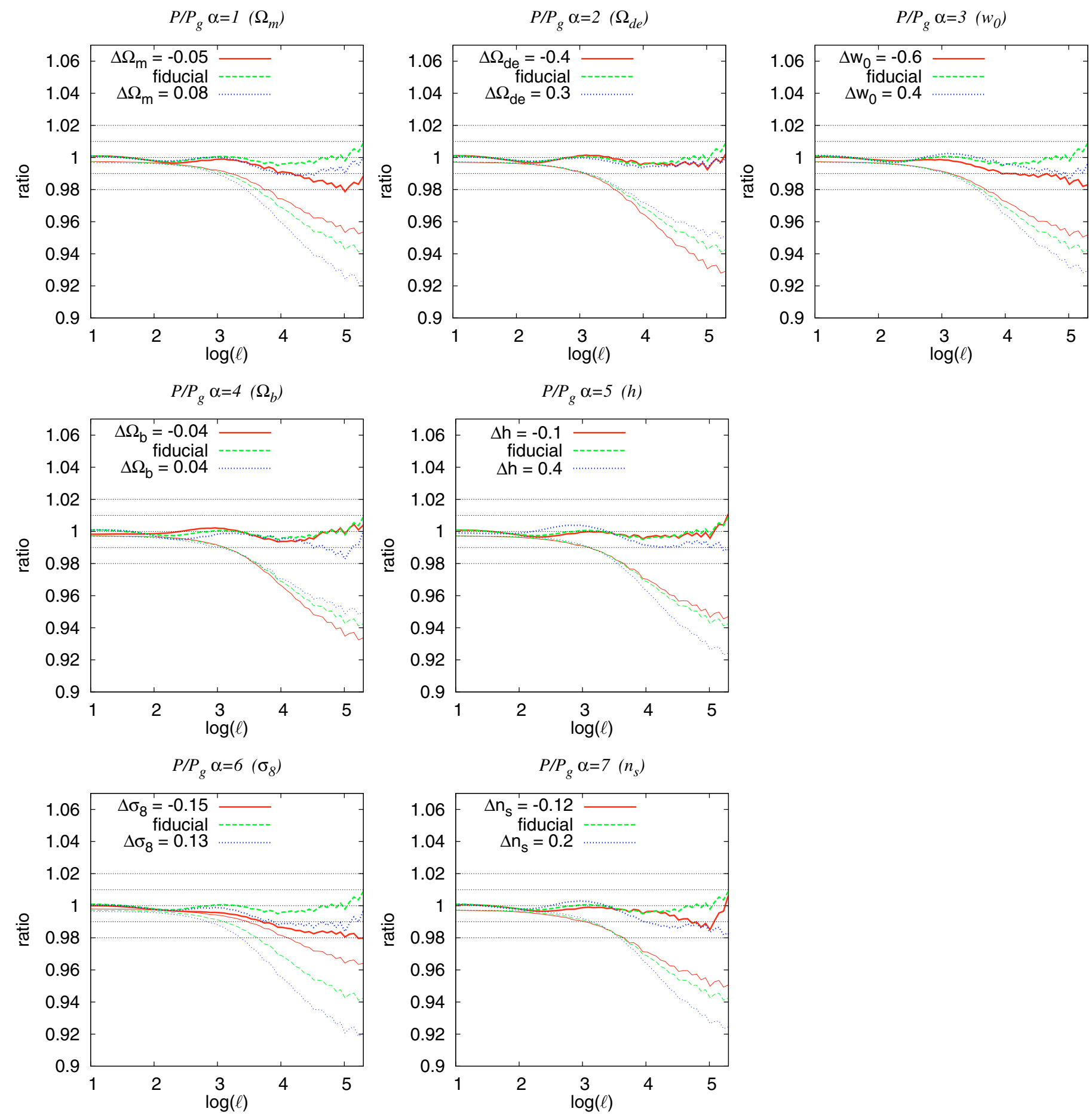

Fig. 3. The fitted reduced-shear power spectrum (thick lines) and the uncorrected convergence power spectrum (thin lines). Both quantities are plotted as ratios with respect to the reduced-shear power spectrum (6), where the first-order correction $P_{\mathrm{g}}^{(1)}$ has been obtained by numerical integration of Eq. (12). In each panel, we show the power spectra at the fiducial model (dashed lines) and the lower (solid) and upper (dashed) boundaries within which a $2 \%$ accuracy is reached for $\ell<2 \times 10^{5}$, see Table 1 .

In Fig. 3, we the plot the ratio of the reduced-shear power spectrum derived using our fitting functions to that obtained by numerical integration of Eq. (12). This is compared to the case of no correction for reduced-shear, corresponding to just the convergence power spectrum. In this latter power spectrum, a downward bias is evident, since the power is underestimated. This bias increases from $1 \%$ at $\ell=1000$ to $6 \%$ at $\ell=10^{5}$ for the fiducial cosmology. In models with more structure, this bias is larger, e.g., $8 \%$ for $\sigma_{8}=0.93$. In contrast, our fitted correction is accurate to better than $1 \%$ for $\ell=2 \times 10^{5}$ at the fiducial point.

We test different redshift distributions by changing to $z_{0}=$ 0.3 and $z_{0}=0.7$, corresponding to mean redshifts of 0.45 and 1 , respectively, and also a redshift bin of width 0.1 around $z=0.75$. In all cases, the fitting formula remains accurate to within $1 \%$ at the fidicual model. 
In Table 1, we denote the parameter ranges for which our fitting formulae are accurate to better than $1 \%$ for $\ell<10^{4}$, and better than $2 \%$ for $\ell<2 \times 10^{5}$.

\section{Conclusions}

The lowest-order correction term for the reduced-shear power spectrum is the dominant contribution from lensing-only effects (Krause \& Hirata 2009). This term is proportional to the third power of the gravitational potential $\phi$, and includes an integral over the lensing bispectrum. In this paper, we have presented fitting formulae for this integral and its derivative with respect to cosmological parameters. This has permitted a more efficient calculation of the reduced-shear correction power spectrum within a parameter range comprising the $68 \%$ confidence region of WMAP7. The fitting scheme reduces the computational effort from tens of minutes for the full integration to a fraction of a second.

For currently available weak-lensing data, the uncertainty in the non-linear power spectrum on small scales is still larger than the bias due to the reduced-shear. For example, the widely-used fitting prescription by Smith et al. (2003) strongly underestimates the power on small scales. More recent numerical simulations however provide fitting formulae that improve the accuracy by a factor 5 to 10 (Heitmann et al. 2008, 2009; Lawrence et al. 2010). Moreover, their emulation scheme can be applied to a wide range of cosmological parameters. This range is comprised in the hypercube for which our fits are valid. In combination, these results provide us with predictions for the weak lensing power spectrum that are accurate to the few percent level.

Acknowledgements. We thank Peter Schneider for helpful comments on the manuscript, and the anonymous referee whose suggestions helped to improve the paper. This research was supported by the DFG cluster of excellence Origin and Structure of the Universe and the Chinese National Science Foundation Nos. 10878003 and 10778725, 973 Program No. 2007CB 815402, Shanghai Science Foundations and Leading Academic Discipline Project of Shanghai Normal University (DZL805).

\section{References}

Bartelmann, M., \& Schneider, P. 2001, Phys. Rep., 340, 297

Bernardeau, F., Van Waerbeke, L., \& Mellier, Y. 1997, A\&A, 322, 1

Dodelson, S., \& Zhang, P. 2005, Phys. Rev. D, 72, 083001

Dodelson, S., Shapiro, C., \& White, M. 2006, Phys. Rev. D, 73, 023009

Eisenstein, D. J., \& Hu, W. 1998, ApJ, 496, 605

Hamana, T. 2001, MNRAS, 326, 326

Heitmann, K., Higdon, D., White, M., et al. 2009, ApJ, 705, 156

Heitmann, K., White, M., Wagner, C., Habib, S., \& Higdon, D. 2008, ApJ, 715, 104

Kaiser, N. 1992, ApJ, 388, 272

Komatsu, E., Smith, K. M., Dunkley, J., et al. 2010, ApJS, submitted, [arXiv: 1001.4538$]$

Krause, E., \& Hirata, C. M. 2009, A\&A, accepted, [arXiv: 0910.3786]

Lawrence, E., Heitmann, K., White, M., et al. 2010, ApJ, 713, 1322

Limber, D. N. 1953, ApJ, 117, 134

Ménard, B., Hamana, T., Bartelmann, M., \& Yoshida, N. 2003, A\&A, 403, 817

Refregier, A., Amara, A., Kitching, T., \& Rassat, A. 2008, A\&A, submitted, [arXiv: 0810.1285]

Schmidt, F., Rozo, E., Dodelson, S., Hui, L., \& Sheldon, E. 2009a, ApJ, 702, 593 Schmidt, F., Rozo, E., Dodelson, S., Hui, L., \& Sheldon, E. 2009b, Phys. Rev. Lett., 103, 051301

Schneider, P., Van Waerbeke, L., Jain, B., \& Kruse, G. 1998, MNRAS, 296, 873 Schneider, P., Van Waerbeke, L., \& Mellier, Y. 2002, A\&A, 389, 729

Schrabback, T., Hartlap, J., Joachimi, B., et al. 2010, A\&A, 516, A63

Scoccimarro, R., \& Couchman, H. M. P. 2001, MNRAS, 325, 1312

Seitz, S. 1993, in Liege International Astrophysical Colloquia, ed. J. Surdej, D. Fraipont-Caro, E. Gosset, S. Refsdal, \& M. Remy, 31, 579 Shapiro, C. 2009, ApJ, 696, 775

Smith, R. E., Peacock, J. A., Jenkins, A., et al. 2003, MNRAS, 341, 1311

White, M. 2005, Astropart. Phys., 23, 349

Wyithe, J. S. B., Winn, J. N., \& Rusin, D. 2003, ApJ, 583, 58

Page 7 is available in the electronic edition of the journal at http://www . aanda.org 
M. Kilbinger: Fitting formulae of the reduced-shear power spectrum $(R N)$

\section{Appendix A: Fitting matrices}

The matrices $B^{(\alpha)}$ and $C^{(\alpha)}(22), a=0, \ldots, M$ contain the coefficients of the reduced-power spectrum fits given in Eq. (21). Here, we provide the numerical values from our fits. The index $\alpha=0$ corresponds to the function $Q$ (12) in the fiducial cosmology, and $\alpha=1, \ldots, 7$ to its derivatives with respect to cosmological parameters (see Table 1). The matrices are also available in electronic form with an example code ${ }^{5}$.

$B^{(0)}=\left(\begin{array}{llll}1.2157 & -1.7061 & 3.613 & -2.588 \\ 22.054 & 14.525 & -10.134 & -1.2063 \\ -1.7221 & -4.2894 & 6.9843 & -3.7669 \\ 41.719 & 34.891 & -40.957 & 6.3019\end{array}\right) ;$

$B^{(1)}=\left(\begin{array}{llll}1.2963 & -2.2435 & 4.7574 & -3.4183 \\ 24.802 & 15.438 & -12.793 & 0.30538 \\ -0.42199 & -14.769 & 28.968 & -16.938 \\ 62.035 & -22.702 & -10.113 & 15.12\end{array}\right)$

$B^{(2)}=\left(\begin{array}{lllll}1.2314 & -1.826 & 3.9322 & -2.9291 \\ 27.286 & -8.0841 & 21.999 & -18.426 \\ -3.2808 & 4.3912 & -6.464 & 2.8446 \\ 62.447 & -73.471 & 122.62 & -71.766\end{array}\right) ;$

$B^{(3)}=\left(\begin{array}{llll}1.1772 & -1.523 & 3.3478 & -2.6269 \\ 17.16 & 39.066 & -56.363 & 24.476 \\ 1.7985 & -17.673 & 25.431 & -12.429 \\ -1.2271 & 192.08 & -259.8 & 113.95\end{array}\right)$;

$B^{(4)}=\left(\begin{array}{llll}1.1983 & -1.5973 & 3.3941 & -2.4472 \\ 21.942 & 28.174 & -32.269 & 10.746 \\ -4.4314 & 9.5715 & -14.092 & 6.4373 \\ 75.097 & -124.48 & 200.67 & -109.85\end{array}\right)$

$B^{(5)}=\left(\begin{array}{llll}1.3643 & -2.6937 & 5.6748 & -4.0482 \\ 22.681 & 17.078 & -13.713 & 0.12838 \\ -1.6597 & -3.5025 & 5.6229 & -3.2007 \\ 44.397 & 11.923 & -3.5699 & -10.617\end{array}\right)$;

$B^{(6)}=\left(\begin{array}{llll}1.2149 & -1.7012 & 3.6033 & -2.5827 \\ 24.779 & 8.4417 & -0.27702 & -6.3675 \\ -2.5782 & -0.084018 & 0.53439 & -0.56922 \\ 53.073 & -11.638 & 28.888 & -27.294\end{array}\right)$;

$B^{(7)}=\left(\begin{array}{llll}1.3469 & -3.3556 & 6.6862 & -4.5164 \\ 25.294 & 6.7075 & 4.1411 & -10.121 \\ -1.0794 & -6.1604 & 9.2899 & -4.7514 \\ 37.736 & 49.648 & -61.197 & 16.44\end{array}\right)$.

$C^{(0)}=\left(\begin{array}{llll}0.56428 & 2.3001 & -3.9649 & 2.427 \\ 0.12548 & -0.94677 & 2.59 & -2.04 \\ 0.1557 & -0.65321 & 0.89795 & -0.58724 \\ -0.063989 & 0.43324 & -1.0211 & 0.7215 \\ 0.0087569 & -0.093102 & 0.24571 & -0.1693 \\ -0.00055709 & 0.008454 & -0.022712 & 0.015138 \\ 1.4809 \cdot 10^{-5} & -0.000274290 .00072501 & -0.00046762\end{array}\right)$

$$
C^{(1)}=\left(\begin{array}{llll}
-0.43501 & -3.1459 & 5.6111 & -3.4686 \\
-0.087793 & 0.80516 & -2.761 & 2.4422 \\
-0.10227 & 0.33267 & -0.18646 & 0.1432 \\
0.073105 & -0.5126 & 1.2891 & -0.95999 \\
-0.023766 & 0.19417 & -0.48231 & 0.33371 \\
0.0030621 & -0.024925 & 0.059059 & -0.039071 \\
-0.0001238 & 0.00098714 & -0.0022652 & 0.0014607
\end{array}\right)
$$

$$
C^{(2)}=\left(\begin{array}{llll}
-0.49063 & -2.7133 & 4.5927 & -2.7656 \\
0.13912 & -0.76984 & 0.7525 & 0.11512 \\
-0.22771 & 1.0278 & -1.3875 & 0.79182 \\
-0.00013136 & -0.029954 & 0.26883 & -0.30505 \\
0.01479 & -0.046285 & -0.0031371 & 0.04091 \\
-0.0020694 & 0.0066385 & -0.0028282 & -0.0018872 \\
8.0142 \cdot 10^{-5} & -0.00026039 & 0.00016485 & 1.1648 \cdot 10^{-5}
\end{array}\right)
$$

$$
C^{(3)}=\left(\begin{array}{llll}
0.25229 & 4.0227 & -6.6135 & 3.7371 \\
-0.52388 & 3.3599 & -5.5903 & 2.5675 \\
0.35958 & -1.7899 & 2.4962 & -1.27 \\
0.076671 & -0.50746 & 0.7332 & -0.2437 \\
-0.045615 & 0.2431 & -0.33587 & 0.13382 \\
0.0052982 & -0.026584 & 0.03616 & -0.014855 \\
-0.00018401 & 0.0008918 & -0.0012 & 0.00049882
\end{array}\right) \text {; }
$$

$C^{(4)}=\left(\begin{array}{llll}0.12913 & 4.7128 & -8.0056 & 4.5759 \\ 0.13963 & -0.11394 & 0.61465 & -0.97735 \\ 0.26098 & -1.3324 & 1.9954 & -1.1216 \\ -0.11282 & 0.48918 & -0.99176 & 0.70867 \\ 0.016542 & -0.075864 & 0.19759 & -0.1533 \\ -0.0010708 & 0.0055907 & -0.017359 & 0.0139 \\ 2.6106 \cdot 10^{-5} & -0.000159160 .00055278 & -0.00044773\end{array}\right)$

$$
C^{(5)}=\left(\begin{array}{llll}
-0.31944 & -3.8608 & 6.7541 & -4.0259 \\
-0.010529 & -0.63367 & 1.0269 & -0.067878 \\
-0.24657 & 1.3721 & -2.1149 & 1.1977 \\
0.051632 & -0.14509 & 0.34221 & -0.34002 \\
0.0010064 & -0.030306 & 0.0014399 & 0.042272 \\
-0.00070106 & 0.0052506 & -0.0026698 & -0.0029094 \\
3.3959 \cdot 10^{-5} & -0.00020532 & 0.00010945 & 8.9968 \cdot 10^{-5}
\end{array}\right)
$$

$$
C^{(6)}=\left(\begin{array}{llll}
0.58241 & 2.2178 & -3.8312 & 2.3591 \\
0.15574 & -1.1301 & 3.0374 & -2.3593 \\
0.1333 & -0.53689 & 0.68283 & -0.46414 \\
-0.073256 & 0.48689 & -1.1436 & 0.80567 \\
0.012694 & -0.11623 & 0.29589 & -0.20194 \\
-0.00098447 & 0.011061 & -0.028339 & 0.018735 \\
2.9427 \cdot 10^{-5} & -0.00036705 & 0.00092519 & -0.0005942
\end{array}\right) ;
$$

$$
C^{(7)}=\left(\begin{array}{llll}
-0.45031 & -3.1685 & 5.4862 & -3.2435 \\
0.088613 & -0.95773 & 1.3824 & -0.2107 \\
-0.20975 & 1.2013 & -1.8198 & 1.0069 \\
0.022754 & -0.030319 & 0.17275 & -0.24886 \\
0.0067345 & -0.052022 & 0.030858 & 0.028623 \\
-0.0011801 & 0.0070072 & -0.004861 & -0.0020472 \\
4.8691 \cdot 10^{-5} & -0.00025807 & 0.0001707 & 6.9607 \cdot 10^{-5}
\end{array}\right) .
$$

5 http://www2.iap.fr/users/kilbinge/reduced 